\title{
Dynamic Shear Suppression in Quantum Phase Space
}

\author{
Maxime Oliva and Ole Steuernagel* \\ School of Physics, Astronomy and Mathematics, University of Hertfordshire, Hatfield AL10 9AB, United Kingdom
}

(Received 12 February 2018; revised manuscript received 18 July 2018; published 18 January 2019)

\begin{abstract}
Classical phase space flow is inviscid. Here we show that in quantum phase space Wigner's probability current $\mathbf{J}$ can be effectively viscous. This results in shear suppression in quantum phase space dynamics which enforces Zurek's limit for the minimum size scale of spotty structures that develop dynamically. Quantum shear suppression is given by gradients of the quantum terms of J's vorticity. Used as a new measure of quantum dynamics applied to several evolving closed conservative 1D bound state systems, we find that shear suppression explains the saturation at Zurek's scale limit and additionally singles out special quantum states.
\end{abstract}

DOI: 10.1103/PhysRevLett.122.020401

The differences between quantum and classical evolution are best investigated in phase space [1]. It is known that quantum evolution in phase space does not obey Liouville's theorem of volume conservation [2,3] and that there is no velocity field in quantum phase space [3] (and therefore no flow). It is less clear why there is no quantum chaos [1,4-7].

Here we show that the effective viscosity of quantum phase space dynamics entails a shear suppression mechanism that fundamentally differentiates quantum from classical dynamics. This observation explains, amongst other things, the absence of quantum chaos.

We consider dynamics in 1D closed conservative systems with spatial coordinate $x$ and momentum $p$. As initial states we use displaced Gaussians $W_{0}\left(x, p, x_{0}, p_{0}\right)=$ $(\pi \hbar)^{-1} \exp \left[-\left(x-x_{0}\right)^{2}-\left(p-p_{0}\right)^{2} / \hbar^{2}\right]$, which are positive [8] and therefore "classical"; $\hbar=h / 2 \pi$ is Planck's constant. After sufficient time $t$ under classical evolution, such a nonsingular initial distribution typically forms thinly stretched out threads, see Fig. 1(c). Generally, structures of $\rho$ become progressively finer as time progresses [9], particularly chaotic systems develop very fine structures quickly [9-12].

Wigner's quantum phase space distribution $W(x, p, t)$ $[13,14]$ is the closest quantum analog $[3,9,14-16]$ of the classical phase space distribution $\rho$. Quantum evolution creates negative regions (blue, delineated by dashed lines at $W=0$, see Fig. 1) (in all figures atomic units $\hbar=1$ and $M=1$ are used [17]). These negative regions represent the existence of quantum coherences, see Refs. [3,9,16,21] and [17].

Interference in phase space [22] is a property built into quantum phase space functions, such as $W$, through the Wigner-Moyal mappings [2,13] between Hilbert space operators and their quantum phase space images [23,24]. This interference limits the fineness of spotty structures that $W$ can have to Zurek's phase space area scale [9]

$$
a_{Z}=\frac{h}{P} \frac{h}{L}=\frac{2 \pi}{K_{x}} \frac{2 \pi}{K_{p}}
$$

[see Figs. 1(b) and 1(d)]. Here length $L$ and momentum $P$ are $W$ 's spread in phase space and thus the area $L P$ (measured in units of action) to which it is confined. The maximal wave numbers associated with $W$ 's structures in $x$ and $p$ are, respectively, $K_{x}=P / \hbar$ and $K_{p}=L / \hbar$ [9] (see Ref. [26] for exceptions). Over time, states develop spotty structures that saturate on the Zurek scale $a_{Z}$ [9].

Here we show that the adherence to Zurek's scale limit in the evolution is best understood in terms of the viscosity [17] of the Wigner current $\mathbf{J}[3,13,27,28]$.

$\mathbf{J}$ is the quantum analog of the classical phase space current $\mathbf{j}=\rho \mathbf{v}$, which transports the probability density $\rho(x, p, t)$ according to Liouville's continuity equation $\partial_{t} \rho=-\boldsymbol{\nabla} \cdot \mathbf{j}$. Here $\mathbf{v}$ is the classical phase space velocity $\mathbf{v}=\left[p / M,-\partial_{x} V(x)\right], M$ the mass of the particle, and $V(x)$ the potential, and $\boldsymbol{\nabla}=\left(\partial_{x}, \partial_{p}\right)$ the gradient operator.

Over time $\rho$ gets sheared since $\mathbf{v}$ creates nonzero gradients of its angular velocity across energy shells. The classical Hamiltonian phase space flow is inviscid as $\mathbf{v}$ is independent of $\rho$. Thus no terms suppress the effects of the angular velocity gradients, and so, as time progresses, nonsingular probability distributions in phase space get sheared into ever finer filaments [see Fig. 1(c)].

We define classical phase space shear as [17]

$$
s(x, p ; H)=\partial_{\hat{\mathbf{v}}_{H}}(-\boldsymbol{\nabla} \times \mathbf{v})=\partial_{\hat{\mathbf{v}}_{H}}\left(\partial_{p} v_{x}-\partial_{x} v_{p}\right),
$$

using the directional derivative across energy shells $\partial_{\hat{\mathbf{v}}_{H}}$, formed from the normalized gradient $\hat{\boldsymbol{\nabla}}_{H}=\boldsymbol{\nabla} H /|\boldsymbol{\nabla} H|$ of the Hamiltonian $H=\left[p^{2} /(2 M)\right]+V(x)$.

The sign convention with the negative curl in $s$ in Eq. (2) was chosen to yield a positive sign for clockwise orientated fields since this is the prevailing direction of the classical 

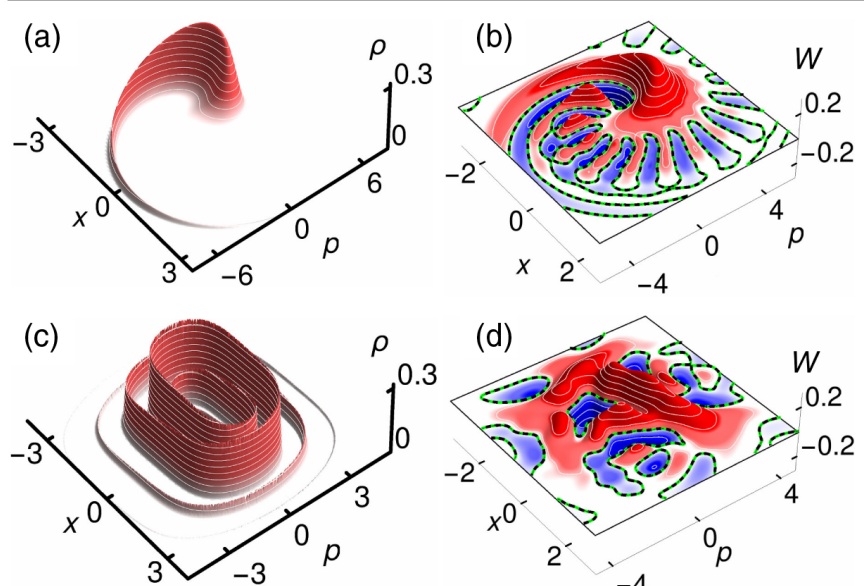

(d)

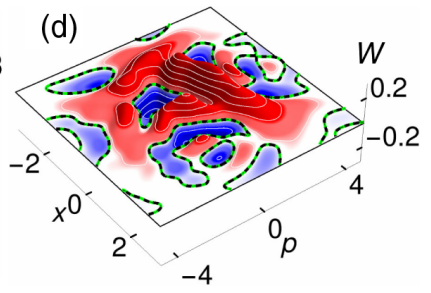

FIG. 1. Comparison between classical and quantum distributions in phase space. For short times quantum evolution resembles classical evolution, compare (b) to (a). But for long times, since quantum evolution creates less fine structures than classical evolution $[9,25]$, their outcomes differ very substantially, contrast (d) with (c). A weakly excited initial state $W_{0}(x, p, 1.5,0)$ is propagated in the soft potential $V_{\mathrm{V}}=31 x^{2} / 10-x^{4} / 81$ for time $t=50$, under, (a), classical evolution and, (b), quantum evolution. Similarly, the state $W_{0}(x, p, 2,0)$ is propagated in the hard potential $V_{\mathrm{U}}=(x / 2)^{4}$ for time $t=25$, under, (c), classical and, (d), quantum evolution.

velocity field $\mathbf{v}$. This choice yields $s>0$ for hard potentials (potentials for which the magnitude of the force increases with increasing amplitude), since they induce clockwise shear, see Fig. 1(c). $s=0$ for harmonic oscillators and free particles, and $s<0$ for soft potentials (potentials for which the magnitude of the force decreases with increasing amplitude), since they induce anticlockwise shear, see Fig. 1(a).

$W^{\prime}$ s evolution is governed by the quantum continuity equation $[3,13]$

$$
\partial_{t} W=-\boldsymbol{\nabla} \cdot \mathbf{J}=-\partial_{x} J_{x}-\partial_{p} J_{p}
$$

Wigner's current $\mathbf{J}$ does not factorize like $\mathbf{j}=\rho \mathbf{v}$ [3]. It has an integral representation $[13,17,28]$. If the potential $V(x)$ is smooth such that it can be expanded into a Taylor series, the integral for $\mathbf{J}$ can be determined explicitly as $[2,13,29]$

$$
\begin{aligned}
\mathbf{J}(x, p, t) & =\mathbf{j}+\mathbf{J}^{Q}=W \mathbf{v}+\left(\begin{array}{c}
0 \\
J_{p}-j_{p}
\end{array}\right) \\
& =W\left(\begin{array}{c}
\frac{p}{M} \\
-\partial_{x} V
\end{array}\right)+\left(\begin{array}{c}
0 \\
-\sum_{l=1}^{\infty} \frac{(i \hbar / 2)^{2 l}}{(2 l+1) !} \partial_{p}^{2 l} W \partial_{x}^{2 l+1} V
\end{array}\right) .
\end{aligned}
$$

J's zeroth-order term in $l$ is the classical term $\mathbf{j}=W \mathbf{v}$ (e.g., $\left.J_{p}\right|_{l=0}=-W \partial_{x} V$ ). Terms of order $l \geq 1$ are the quantum correction terms $\mathbf{J}^{Q}=\mathbf{J}-\mathbf{j}$. They are only
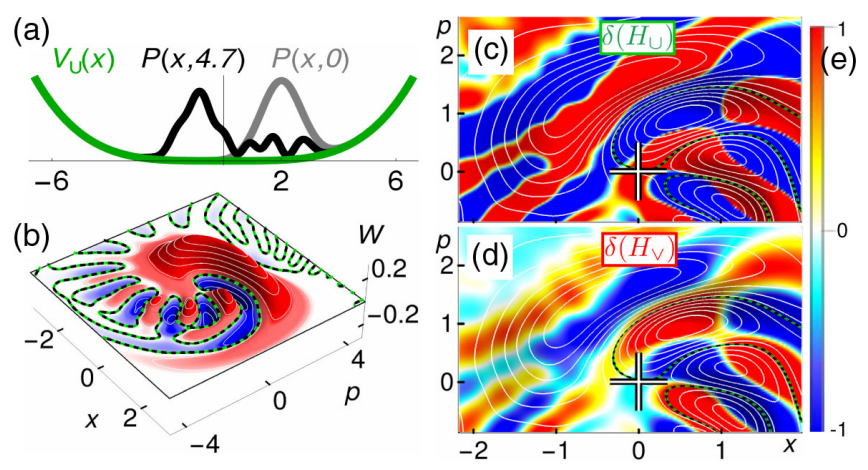

FIG. 2. Polarization of the vorticity $\delta$ and inversion of this polarization. The comparison between (c) and (d) shows polarization inversion. (a) sketch of hard potential $V_{\mathrm{U}}=$ $(x / 2)^{4}$ together with probability distributions $P(x, t)=$ $|\Psi(x, t)|^{2}$ (black curve) of state evolved in $V_{U}$ from initial state $W_{0}(x, p, 2,0)$ (grey curve). $W$ for $t=4.7$ in (a) is shown in (b). White contours of $W$ [the origin $(x, p)=(0,0)$ is labeled by a white cross] are overlaid with colors [legend given in sidebar (e)] representing values of $\tanh \left[50 \delta\left(H_{\mathrm{U}}\right)\right]$, (c), and $\tanh \left[50 \delta\left(H_{\mathrm{V}}\right)\right]$, (d). For the Hamiltonians $H_{\mathrm{V}}$ and $H_{\mathrm{U}}$ the same potentials as in Fig. 1 are used.

present for anharmonic potentials [28], which is why only anharmonic potentials create coherences. Harmonic systems' phase space dynamics is classical $[3,17,28]$.

The reaction of quantum dynamics to classical shear $s$ has to reside in $\mathbf{J}^{Q}$. To extract it we form the vorticity $\delta$ of $\mathbf{J}^{Q}$

$$
\delta(x, p, t ; H)=-\nabla \times \mathbf{J}^{Q}=\partial_{p} J_{x}^{Q}-\partial_{x} J_{p}^{Q} .
$$

$\delta$ 's sign distribution shows a pronounced polarization pattern, see for example Fig. 2(c): specifically, on the positive main ridge of $W$ [Fig. 2(b)] $\delta$ tends to be positive on the inside (towards the origin) and negative on the outside. Because of this, the outside is being slowed down while the inside speeds up. This polarized distribution of $\delta$ therefore counteracts the classical shear $\left(s_{V_{U}}>0\right)$ and can suppress it altogether.

The same applies to other positive regions of $W$, whereas for its negative regions the current $\mathbf{J}$ tends to be inverted $[27,30]$ just as $\delta$ 's polarization pattern.

Switching the governing potential from hard, $V_{\mathrm{U}}$, to soft, $V_{\mathrm{V}}$ (using the same state but different dynamics), reverses the classical shear, see Fig. 1. Accordingly, a reversal of the polarization pattern of Fig. 2(c) occurs in Fig. 2(d).

The distribution of $\delta$ 's polarization can be picked up with the directional derivative $\partial_{\hat{\mathbf{v}}_{H}} \delta(t ; H)$. This we multiply with $W$, because negative regions of $W$ invert the current $\mathbf{J}$ [27], and because we want to weight it with the local contribution of the state. The resulting measure for weighted shear polarization is $\pi(x, p, t ; H)=W(t) \partial_{\hat{\mathbf{v}}_{H}} \delta(t ; H)$. Its average across phase space is $W$ 's shear polarization 

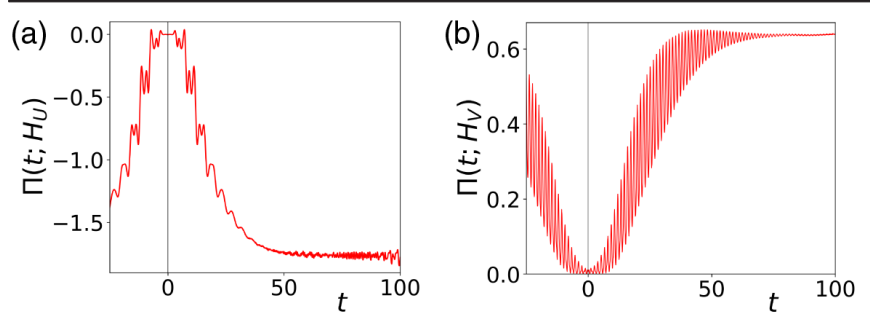

FIG. 3. $\Pi(t ; H)$ levels off over time as systems saturate. $\Pi$ 's time evolution for, (a), initial state $W_{0}(x, p, 9,0)$, for the hard potential $V_{\mathrm{U}}=x^{4} / 500$, and, (b), $W_{0}(x, p, 3,0)$, for the soft potential $V_{\mathrm{V}}=31 x^{2} / 10-x^{4} / 81$. In accord with our signconvention for Eq. (2) $\Pi\left(t ; H_{\cup}\right)$ drops over time whereas $\Pi\left(t ; H_{\mathrm{V}}\right)$ rises, until the system saturates.

$$
\Pi(t ; H)=\left\langle\langle\pi(t ; H)\rangle=\iint_{-\infty}^{\infty} d x d p \pi(x, p) .\right.
$$

Initially $|\Pi(t)|$ rises on average and after a while levels off and settles, see Fig. 3 .

We emphasize that the leveling-off behavior of $\Pi(t)$ is in marked contrast to the classical case: in simple bound state systems, the states never saturate, instead, for long enough times $\left\langle\left\langle\partial_{\hat{\mathbf{v}}_{H}}(-\boldsymbol{\nabla} \times \mathbf{j})\right\rangle\right\rangle \propto t$ since $\rho(t)$ gets stretched out linearly into ever finer threads, see Fig. 1(c) and Ref. [17]. Also the quantum evolution can shrink structures of $W$ in size, but $W$ 's minimal structures are forced to saturate at the Zurek scale by shear suppression.

When a state saturates, the gradients in the quantum terms of $\mathbf{J}$ become so large that they strongly quantum suppress the classical shear inherent in $\mathbf{J}$. Where minimal structures of $W$ have formed, this quantum shear suppression prevents still finer structures from developing: J's effective viscosity enforces the saturation of states at the Zurek scale.

When this happens, $\Pi(t)$ has settled, see Figs. 3, 4, and 6.

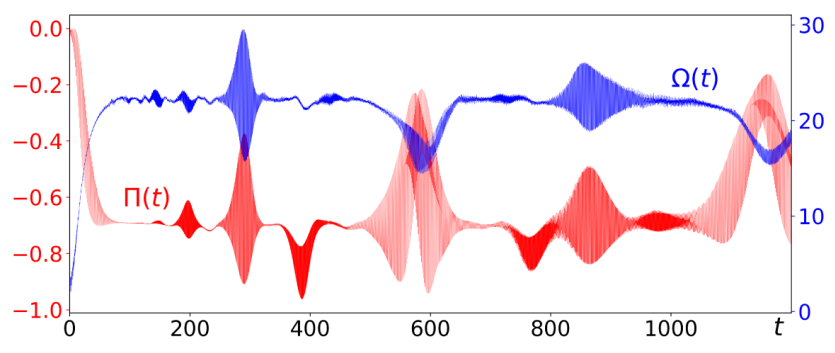

FIG. 4. Shear suppression $\Pi(t)$ and frequency contents $\Omega(t)$ can evolve similarly. A Gaussian state $W_{0}(x, p, 3,0)$ is evolved in a hard potential $V_{U}=31 x^{2} / 10+x^{4} / 81$. While $\Omega(t)$ rises (here, $\left.\Omega_{\max }=182.5\right)$, as $W$ develops fine structure, $\Pi(t)$ drops, since we consider a hard potential $V_{\mathrm{U}}$. Both curves $\Pi(t)$ and $\Omega(t)$ level off at the time where $W$ 's fine structures saturate at the Zurek scale. Here the oscillations around the respective mean values for saturated systems are due to the formation of special (partial revival) states [31,32], for details, see Fig. 6 and Ref. [17].
To make explicit the connection between shear suppression $\Pi(t)$ and the saturation of systems at the Zurek scale we define $W$ 's spatial frequency contents $\Omega$ as

$$
\Omega(t)=\frac{\iint d k_{x} d k_{p}\left|\tilde{\widetilde{W}}\left(k_{x}, k_{p}, t\right) k_{x} k_{p}\right|}{\iint d k_{x} d k_{p}\left|\tilde{W}\left(k_{x}, k_{p}, t\right)\right|}<2 K_{X} K_{P},
$$

where $\tilde{\widetilde{W}}\left(k_{x}, k_{p}\right)$ is the 2D Fourier transform of $W(x, p)$. Since a state cannot only consist of structures at the Zurek scale, Eq. (8) obeys the inequality $\Omega<\Omega_{\max }=\left[\left(8 \pi^{2}\right) / a_{z}\right]=$ $2 K_{X} K_{P}$, compare Eq. (1) and Ref. [17].

Figure 4 demonstrates that for simple systems changes of the shear polarization $\Pi(t)$ can go hand in hand with those of the spatial frequency contents $\Omega(t)$. This establishes that shear suppression constitutes the mechanism by which quantum dynamics conforms with interference in phase space.

Interestingly, both measures single out special states: those states for which the values of $\Pi$ and $\Omega$ deviate from the typical saturated system states' values. In the case of weakly excited single well bound state systems the special states happen to be partial-revival states [31,32], see Fig. 6. Some details of Fig. 4 can be understood from the observation that even partial-revival states feature more symmetric interference patterns, which lowers their frequency contents $\Omega$, when compared with odd partialrevival states.

We emphasize that $\Pi$ and $\Omega$ can measure aspects of the dynamics very differently from each other, see Ref. [17] for an illustration.

The spectrum $\tilde{\Pi}(\omega)$ of $\Pi(t)$ is smoother than the spectrum $\tilde{\Omega}(\omega)$ of $\Omega(t)$, see Fig. 5. Valuable information is more easily accessible through $\Pi$ than $\Omega$ since this smoothness allows us to cut out frequency bands without sensitive dependence on the cut location. Additionally, $\Pi$ provides information more readily than the typically used wave function overlap $\mathcal{P}(t)=\left|\left\langle\Psi_{0} \mid \Psi(t)\right\rangle\right|^{2}$. This is because $\mathcal{P}(t)$ depends sensitively on its initial state $\Psi_{0}$, but also because the spectrum of $\mathcal{P}(t)$ is noisier and does not

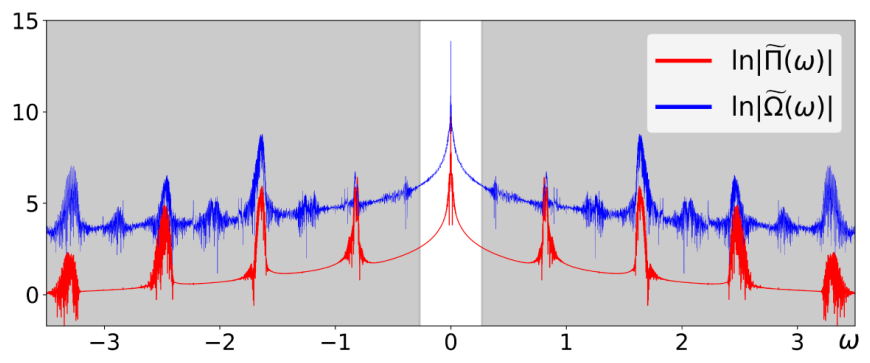

FIG. 5. Fourier spectra $\tilde{\Pi}(\omega)$ and $\tilde{\Omega}(\omega)$ of the time series $\Pi(t)$ and $\Omega(t)$ in Fig. 4. Note that $\Pi(t)$ provides a smoother spectrum than $\Omega(t)$. Cutting out $\tilde{\Pi}$ 's central band ( $\tilde{\Pi}_{0}$ within white corridor) allows us to smooth $\Pi(t)$; see Fig. 6 . 


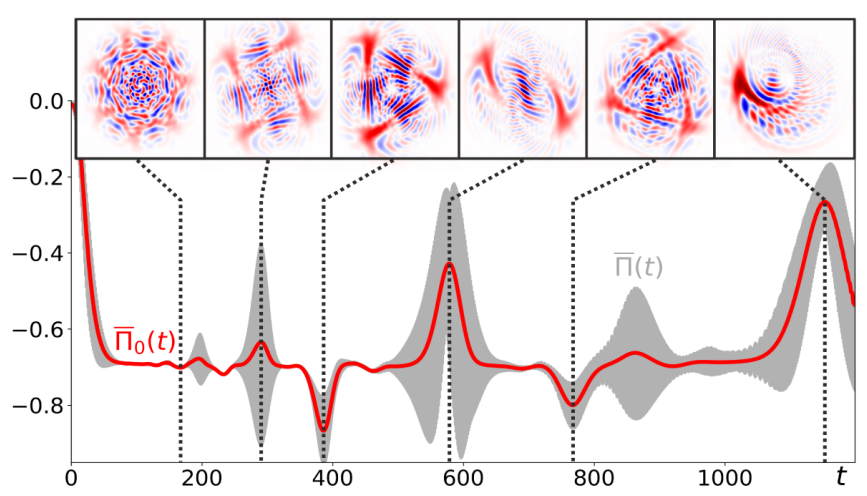

FIG. 6. Smoothed $\Pi(t)$ picks out special states. The inverse Fourier transform of the central band $\tilde{\Pi}_{0}(\omega)$ (highlighted in Fig. 5) yields a (thick red) smoothed curve $\bar{\Pi}_{0}(t)$ of $\Pi(t)$ (gray curve). Deviations of $\bar{\Pi}_{0}(t)$ from the settled value $(\approx-0.7)$ singles out "unsaturated" special states: the evolution shows an approximate recurrence of the initial state at time $T \approx 1154$. Pronounced peaks and troughs at intermediate times identify fractional revival states $[17,31]$ with special $n$-fold symmetries.

have a central peak that provides accessible information in the manner that $\tilde{\Pi}_{0}$ does, see Ref. [17].

Our approach can be applied to a wide range of systems including Kerr systems [33], driven and dissipative systems [34], higher-dimensional continuous systems [13], and discrete spin systems [15,35].

To conclude, quantum dynamics in phase space can be effectively viscous; we have traced this back to the behavior of the quantum corrections in Wigner's phase space current J. Quantum suppression of classical shear generates shear polarization patterns that characterize the difference between quantum and classical phase space dynamics. J's viscosity limits the fineness of structures formed in quantum phase space dynamics. The quantification of shear polarization patterns using $\Pi(t)$ provides new insight into the character of quantum phase space dynamics. Additionally, studying the time series of $\Pi$ we find that it sensitively displays features of the dynamics, picks out special quantum states, does not rely on arbitrarily chosen reference states, can be frequency filtered, and provides information on the dynamics in a robust way.

For the study of the dynamics of continuous quantum systems we expect that the shear suppression polarization $\Pi(t)$ will prove to be a valuable alternative to the wave function overlap probability $\mathcal{P}(t)$.

O. S. thanks Michael Berry for his encouragement to pursue this research and Alan McCall and Martin Hardcastle for their careful reading of the manuscript.

*Ole.Steuernagel@gmail.com

[1] J. Gong and P. Brumer, Chaos and quantum-classical correspondence via phase-space distribution functions, Phys. Rev. A 68, 062103 (2003).
[2] J.E. Moyal, Quantum mechanics as a statistical theory, Proc. Cambridge Philos. Soc. 45, 99 (1949).

[3] M. Oliva, D. Kakofengitis, and O. Steuernagel, Anharmonic quantum mechanical systems do not feature phase space trajectories, Physica (Amsterdam) 502A, 201 (2017).

[4] W. H. Zurek and J.P. Paz, Decoherence, Chaos, and the Second Law, Phys. Rev. Lett. 72, 2508 (1994).

[5] G. Casati and B. V. Chirikov, Comment on Decoherence, Chaos, and the Second Law, Phys. Rev. Lett. 75, 350 (1995).

[6] W. H. Zurek and J. P. Paz, Zurek and Paz Reply, Phys. Rev. Lett. 75, 351 (1995).

[7] I. Gomez and M. Castagnino, On the classical limit of quantum mechanics, fundamental graininess and chaos: Compatibility of chaos with the correspondence principle, Chaos Solitons Fractals 68, 98 (2014).

[8] R. L. Hudson, When is the Wigner quasi-probability density non-negative?, Rep. Math. Phys. 6, 249 (1974).

[9] W. H. Zurek, Sub-Planck structure in phase space and its relevance for quantum decoherence, Nature (London) 412, 712 (2001).

[10] M. V. Berry, Regular and Irregular Motion, AIP Conf. Proc. 46, 16 (1978).

[11] D. D. Nolte, The tangled tale of phase space, Phys. Today 63, No. 4, 33 (2010).

[12] P. Cvitanović, R. Artuso, R. Mainieri, G. Tanner, and G. Vattay, Chaos: Classical and Quantum (2012), http://chaosbook.org.

[13] E. Wigner, On the quantum correction for thermodynamic equilibrium, Phys. Rev. 40, 749 (1932).

[14] M. Hillery, R. F. O'Connell, M. O. Scully, and E. P. Wigner, Distribution functions in physics: Fundamentals, Phys. Rep. 106, 121 (1984).

[15] T. Tilma, M. J. Everitt, J. H. Samson, W. J. Munro, and K. Nemoto, Wigner Functions for Arbitrary Quantum Systems, Phys. Rev. Lett. 117, 180401 (2016).

[16] D. Leibfried, T. Pfau, and C. Monroe, Shadows and mirrors: Reconstructing quantum states of atom motion, Phys. Today 51, No. 4, 22 (1998).

[17] See Supplemental Material at http://link.aps.org/ supplemental/10.1103/PhysRevLett.122.020401 for more detailed considerations, which includes [18-20].

[18] R. P. Feynman, R. Leighton, and M. Sands, The Feynman Lectures on Physics (Addison-Wesley, Reading, 1965).

[19] T. Takabayasi, The formulation of quantum mechanics in terms of ensemble in phase space, Prog. Theor. Phys. 11, 341 (1954).

[20] C. Kurtsiefer, T. Pfau, and J. Mlynek, Measurement of the Wigner function of an ensemble of helium atoms, Nature (London) 386, 150 (1997).

[21] R. P. Feynman, Negative Probability (Routledge, London, New york, 1987), pp. 235-248, http://cds.cern.ch/record/ 154856/files/pre-27827.pdf.

[22] W. P. Schleich, Quantum Optics in Phase Space (WileyVCH, 2001).

[23] C. Rasinariu, Shape invariance in phase space, Fortschr. Phys. 61, 4 (2013).

[24] J. Hancock, M. A. Walton, and B. Wynder, Quantum mechanics another way, Eur. J. Phys. 25, 525 (2004).

[25] M. V. Berry and N. L. Balazs, Evolution of semiclassical quantum states in phase space, J. Phys. A 12, 625 (1979). 
[26] M. Oliva and O. Steuernagel, Structures far below subPlanck scale in quantum phase-space through superoscillations, Phys. Rev. A 95, 052112 (2017).

[27] O. Steuernagel, D. Kakofengitis, and G. Ritter, Wigner Flow Reveals Topological Order in Quantum Phase Space Dynamics, Phys. Rev. Lett. 110, 030401 (2013).

[28] D. Kakofengitis, M. Oliva, and O. Steuernagel, Wigner's representation of quantum mechanics in integral form and its applications, Phys. Rev. A 95, 022127 (2017).

[29] H. J. Groenewold, On the principles of elementary quantum mechanics, Physica (Utrecht) 12, 405 (1946).

[30] D. Kakofengitis and O. Steuernagel, Wigner's quantum phase space flow in weakly-anharmonic weakly-excited two-state systems, Eur. Phys. J. Plus 132, 381 (2017).
[31] I. S. Averbukh and N.F. Perelman, Fractional revivals: Universality in the long-term evolution of quantum wave packets beyond the correspondence principle dynamics, Phys. Lett. A 139, 449 (1989).

[32] R. W. Robinett, Quantum wave packet revivals, Phys. Rep. 392, 1 (2004).

[33] M. Oliva and O. Steuernagel, Quantum Kerr oscillators' evolution in phase space: Wigner current, symmetries, shear suppression and special states, arXiv:1811.02952.

[34] O. D. Friedman and M. P. Blencowe, The Wigner flow for open quantum systems, arXiv:1703.04844.

[35] A. B. Klimov and S. M. Chumakov, On the su (2) Wigner function dynamics, Rev. Mex. Fis. 48, 317 (2002). 